\title{
Analysis, Control, Synchronization and SPICE Implementation of a Novel 4-D Hyperchaotic Rikitake Dynamo System without Equilibrium
}

\author{
S. Vaidyanathan*, ${ }^{*}$, Ch. K. Volos ${ }^{2}$, and V. -T. Pham ${ }^{3}$ \\ ${ }^{I}$ Research and Development Centre, Vel Tech University, Avadi, Chennai-600062, Tamil Nadu, India. \\ ${ }^{2}$ Department of Physics, Aristotle University of Thessaloniki, Thessaloniki, GR-54124, Greece. \\ ${ }^{3}$ School of Electronics and Telecommunications, Hanoi University of Science and Technology, Hanoi, Vietnam.
}

Received 30 September 2014; Revised 15 November 2014; Accepted 29 November 2014

\begin{abstract}
Chaos theory has wide applications and its importance can be seen by the voluminous publications on various applications in several branches of science, commerce and engineering. Control, tracking or regulation and synchronization of different types of chaotic systems are importance areas of research in the control literature and various methods have been adopted over the past few decades for tackling these research problems. Also, the discovery of novel chaotic and hyperchaotic systems in various applications, their qualitative properties and the control of such systems are also important research areas in chaos theory. This paper announces a novel 4-D hyperchaotic Rikitake dynamo system, which is derived by adding a state feedback control to the famous 3-D Rikitake two-disk dynamo system (1958). The frequent and irregular reversals of the Earth's magnetic field inspired a number of early studies involving electrical currents within the Earth's molten core. One of the first such models to exhibit reversals was Rikitake's two-disk dynamo system (Rikitake, 1958). This paper discusses the qualitative properties of the novel hyperchaotic Rikitake dynamo system. We note that the novel hyperchaotic Rikitake dynamo system has no equilibrium points. The Lyapunov exponents of the hyperchaotic Rikitake dynamo system are found as $L_{1}=0.09136, L_{2}=$ $0.02198, L_{3}=0$ and $L_{4}=-2.11190$. The Kaplan-Yorke fractional dimension of the novel hyperchaotic Rikitake dynamo system is found as $D_{K Y}=3.05367$. Next, this paper discusses control and synchronization of the novel hyperchaotic Rikitake dynamo system with unknown parameters using adaptive control method. The main results are established using Lyapunov stability theory and numerically illustrated using MATLAB. Finally, for the 4-D novel hyperchaotic system, an electronic circuit realization in SPICE has been described to confirm the feasibility of the theoretical hyperchaotic Rikitake dynamo model.
\end{abstract}

Keywords: Chaos, chaotic systems, hyperchaos, hyperchaotic systems, adaptive control, chaos synchronization, circuit simulation.

\section{Introduction}

Chaotic systems are defined as nonlinear dynamical systems which are very sensitive to initial conditions, topologically mixing and also with dense periodic orbits [1]. The first 3-D chaotic system was experimentally verified by Lorenz [2].

The sensitivity to initial conditions of a chaotic system is indicated by a positive Lyapunov exponent. If the sum of all Lyapunov exponents is negative, then the chaotic system is called a dissipative system.

Great interest has been shown in the chaos literature in the modelling of many 3-D chaotic systems such as Rössler system [3], Rabinovich system [4], ACT system [5], Sprott

* E-mail address: sundarvtu@gmail.com ISSN: 1791-2377 @ 2015 Kavala Institute of Technology. All rights reserved. systems [6], Chen system [7], Lü system [8], Shaw system [9], Feeny system [10], Shimizu system [11], Liu-Chen system [12], Cai system [13], Tigan system [14], Colpitt's oscillator [15], WINDMI system [16], Zhou system [17], etc.

Recently, many 3-D chaotic systems have been discovered such as Li system [18], Elhadj system [19], Pan system [20], Sundarapandian system [21], Yu-Wang system [22], Sundarapandian-Pehlivan system [23], Zhu system [24], Vaidyanathan systems [25-33], VaidyanathanMadhavan system [34], Pehlivan-Moroz-Vaidyanathan system [35], Pham systems [36-37], etc.

Chaos theory has many important applications in science and engineering such as vibration control [38-40], oscillators [41-43], robotics [44-47], chemical reactors [4850], biology [51,52], ecology [53,54], cardiology [55], memristors [56-58], neural networks [59-61], secure communications [62-65], cryptosystems [66-69], networks 
[70, 71], economics [72-74], market forecasting [75], etc. Several works have been carried out by researchers in exploring new applications of chaos theory in diverse fields.

Chaos control and chaos synchronization are important research problems in the chaos theory. In the last three decades, many mathematical methods have been developed successfully to address these research problems.

The study of control of a chaotic system investigates methods for designing feedback control laws that globally or locally asymptotically stabilize or regulate the outputs of a chaotic system.

Many methods have been developed for the control and tracking of chaotic systems such as active control [76-80], adaptive control [81-87], backstepping control [88-90], sliding mode control [91, 92], etc.

A hyperchaotic system is generally defined as a chaotic system with at least two positive Lyapunov exponents. Thus, the dynamics of a hyperchaotic system are expended in several different directions simultaneously. Thus, the hyperchaotic systems have more complex dynamical behaviour and hence they have miscellaneous applications in engineering such as secure communications [93-95], cryptosystems [96-98], encryption [99-101], electrical circuits [102-105], etc.

Chaos synchronization problem deals with the synchronization of a couple of systems called the master or drive system and the slave or response system. To solve this problem, control laws are designed so that the output of the slave system tracks the output of the master system asymptotically with time.

Because of the butterfly effect, the chaos synchronization problem is seemingly a challenging research problem even when the initial conditions of the master and slave systems are nearly identical because of the exponential divergence of the outputs of the two systems in the absence of any control. The synchronization of chaotic systems has applications in secure communications [106-109], cryptosystems [110,111], encryption [112,113], etc.

In the chaos literature, many different methodologies have been also proposed for the synchronization and antisynchronization of chaotic systems such as PC method [114], active control [115-125], time-delayed feedback control [126,127], adaptive control [128-139], sampled-data feedback control [140-142], backstepping control [143-149], sliding mode control [150-158], etc.

The minimum dimension for an autonomous, continuous-time, hyperchaotic system is four. Since the discovery of the first hyperchaotic system by Rössler in 1979 [159], many 4-D hyperchaotic systems have been reported in the literature such as hyperchaotic Lorenz system [160], hyperchaotic Lü system [161], hyperchaotic Chen system [162], hyperchaotic Wang system [163], hyperchaotic Newton-Leipnik system [164], hyperchaotic Vaidyanathan systems [165-167], etc.

In this research work, a novel 4-D hyperchaotic Rikitake dynamo system has been proposed, which is derived by adding a feedback control to the famous 3-D Rikitake twodisk dynamo system (1958) found by Rikitake [168]. An extensive study by Cook and Roberts (1970) established chaos in the Rikitake two-disk dynamo system [169].

The 4-D novel hyperchaotic Rikitake dynamo system proposed in this research work has the Lyapunov exponents $L_{1}=0.09136, L_{2}=0.02198, L_{3}=0$ and $L_{4}=-2.11190$.

Also, the Kaplan-Yorke dimension of the 4-D hyperchaotic Rikitake dynamo system is found as $D_{K Y}=3.05367$.
It is noted that the novel hyperchaotic Rikitake dynamo system does not have any equilibrium point, which is a novel feature of the system.

Some examples of hyperchaotic system without an equilibrium point are Wang's system [170], Pham's systems $[171,172]$, etc.

According to a new classification of chaotic dynamics $[173,174]$, there are two types of attractors: self-excited attractors and hidden attractors. A self-excited attractor has a basin of attraction that is excited from unstable equilibria. In contrast, hidden attractor cannot be found by using a numerical method in which a trajectory started from a point on the unstable manifold in the neighbourhood of an unstable equilibrium [173]. Studying hyperchaotic systems with hidden attractors is still an open research problem[175,176].

The paper is organized as follows. In Section 2, we describe the equations and phase portraits of the novel hyperchaotic Rikitake dynamo system. In Section 3, we derive the qualitative properties of the novel hyperchaotic Rikitake dynamo system. In Section 4, we derive an adaptive controller for the stabilization of 4-D novel hyperchaotic Rikitake dynamo system with unknown parameters.

In Section 5, we derive an adaptive controller for the synchronization of identical 4-D novel hyperchaotic Rikitake dynamo systems with unknown parameters. Section 6 contains a circuit realization of the proposed 4-D hyperchaotic Rikitake dynamo model. Section 7 concludes this research work with a summary of main results.

\section{A 4-D Novel Hyperchaotic Rikitake Dynamo System}

The frequent and irregular reversals of the Earth's magnetic field inspired a number of early studies involving electrical currents within the Earth's molten core. One of the first such models to report reversals was the Rikitake two-disk dynamo model [168].

The dynamics of the 3-D Rikitake two-disk dynamo system is described by

$$
\left\{\begin{array}{l}
\frac{d x_{1}}{d t}=-a x_{1}+x_{2} x_{3} \\
\frac{d x_{2}}{d t}=-a x_{2}+x_{1}\left(x_{3}-b\right) \\
\frac{d x_{3}}{d t}=1-x_{1} x_{2}
\end{array}\right.
$$

where $x_{1}, x_{2}, x_{3}$ are the states and $a, b$ are constant, positive parameters. We note that the Rikitake dynamo system (1) has the same number of terms as the Lorenz chaotic system [2] but with an additional quadratic nonlinearity.

The parameter $a$ represents resistive dissipation and the parameter $b$ represents the difference in the angular velocities of the two disks.

The Rikitake dynamo system (1) depicts a chaotic attractor when the parameter values are taken as:

$a=1 \quad b=1$

For numerical simulations, we take the initial conditions of the 3-D Rikitake two-disk dynamo system (1) as $x_{1}(0)=0.8, x_{2}(0)=0.2$, and $x_{3}(0)=0.4$.

Also, for the parameter values given in (2), the Lyapunov exponents of the Rikitake dynamo system (1) are calculated as $L_{1}=0.12829, L_{2}=0$ and $L_{3}=-2.12736$. 
The 3-D portrait of the two-scroll chaotic attractor of the Rikitake dynamo system (1) for the parameter values given in (2) is depicted in Fig. 1.

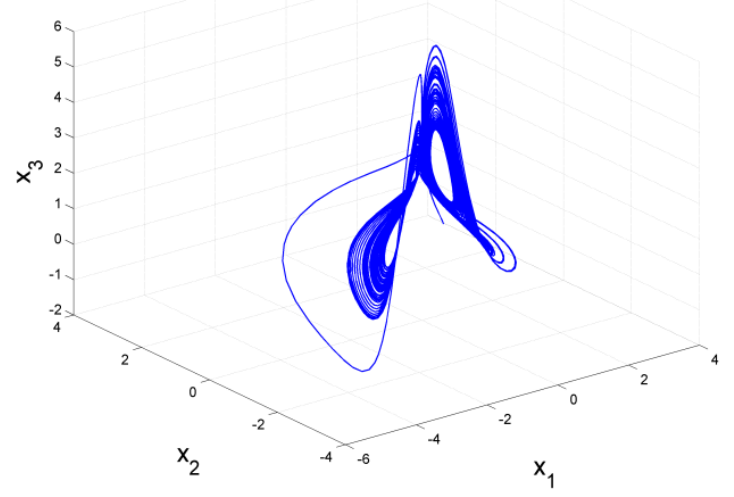

Fig. 1. The 2-scroll attractor of the Rikitake dynamo system in $R^{3}$.

In this work, we derive a novel 4-D hyperchaotic Rikitake dynamo model by adding a state feedback control to the 3-D Rikitake dynamo model (1) as follows.

$$
\left\{\begin{array}{l}
\frac{d x_{1}}{d t}=-a x_{1}+x_{2} x_{3}-p x_{4} \\
\frac{d x_{2}}{d t}=-a x_{2}+x_{1}\left(x_{3}-b\right)-p x_{4} \\
\frac{d x_{3}}{d t}=1-x_{1} x_{2} \\
\frac{d x_{4}}{d t}=c x_{2}
\end{array}\right.
$$

where $x_{1}, x_{2}, x_{3}, x_{4}$ are the state variables and $a, b, c, p$ are constant, positive, parameters.

The 4-D system (3) is hyperchaotic when the parameter values are taken as

$a=1, b=1, c=0.7, p=1.7$

For numerical simulations, we take the initial values of the 4 -D system $(3)$ as $x_{1}(0)=0.8, x_{2}(0)=0.2, x_{3}(0)=0.4$ and $x_{4}(0)=0.6$.

The Lyapunov exponents of the 4-D system (3) are calculated as:

$$
\left\{\begin{array}{llc}
L_{1}= & 0.09136 \\
L_{2}= & 0.02198 \\
L_{3}= & 0 \\
L_{4}= & -2.11190
\end{array}\right.
$$

Thus, the 4-D system (3) has two positive Lyapunov exponents, which shows that the system is hyperchaotic. Hence, the hyperchaotic Rikitake dynamo system (3) has very complex dynamics.

The Kaplan-Yorke dimension of the hyperchaotic Rikitake system (5) is obtained as:

$$
D_{K Y}=3+\frac{L_{1}+L_{2}+L_{3}}{\left|L_{4}\right|}=3.05367
$$

Figures 2-5 depict the 3-D phase portraits of the 4-D hyperchaotic Rikitake dynamo system (3) in $\left(x_{1}, x_{2}, x_{3}\right)$, $\left(x_{1}, x_{2}, x_{4}\right),\left(x_{1}, x_{3}, x_{4}\right)$, and $\left(x_{2}, x_{3}, x_{4}\right)$ spaces, respectively.

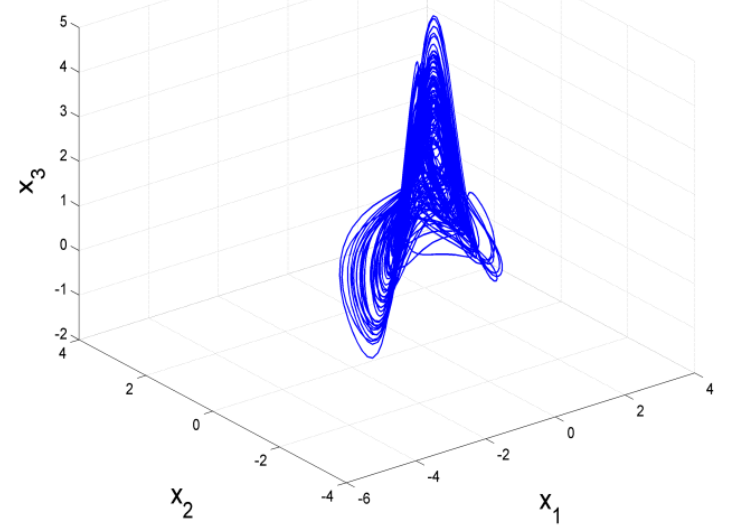

Fig. 2. The 3-D projection of the hyperchaotic Rikitake dynamo system on the $\left(x_{1}, x_{2}, x_{3}\right)$ space.

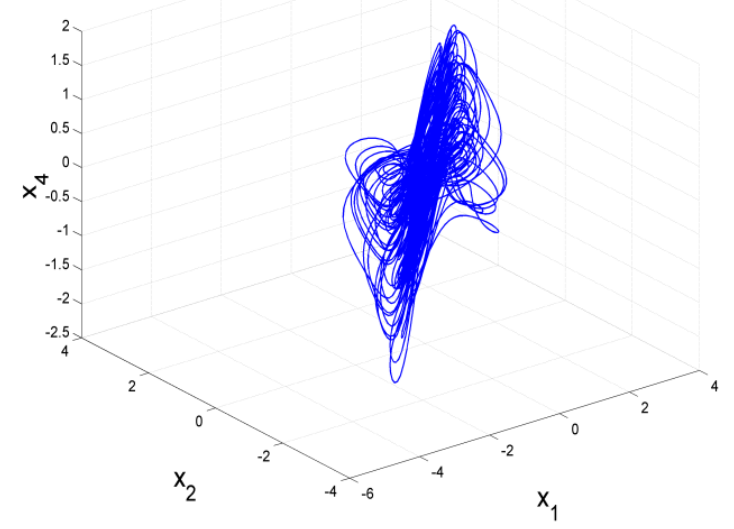

Fig. 3. The 3-D projection of the hyperchaotic Rikitake dynamo system on the $\left(x_{1}, x_{2}, x_{4}\right)$ space.

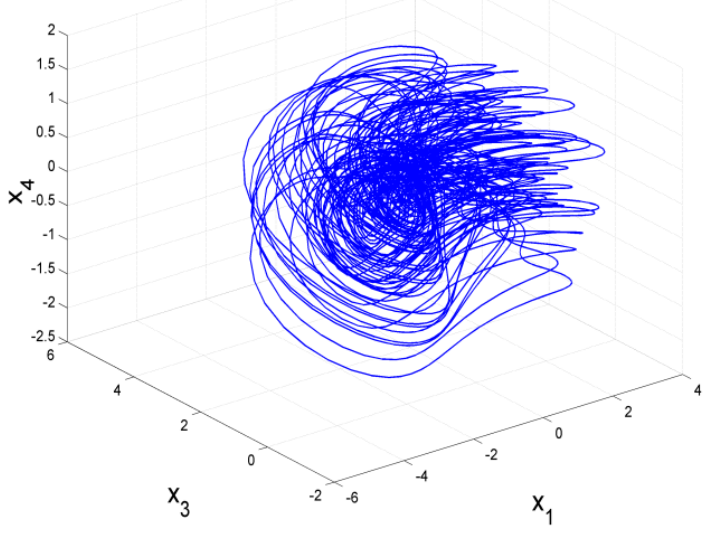

Fig. 4. The 3-D projection of the hyperchaotic Rikitake dynamo system on the $\left(x_{1}, x_{3}, x_{4}\right)$ space. 


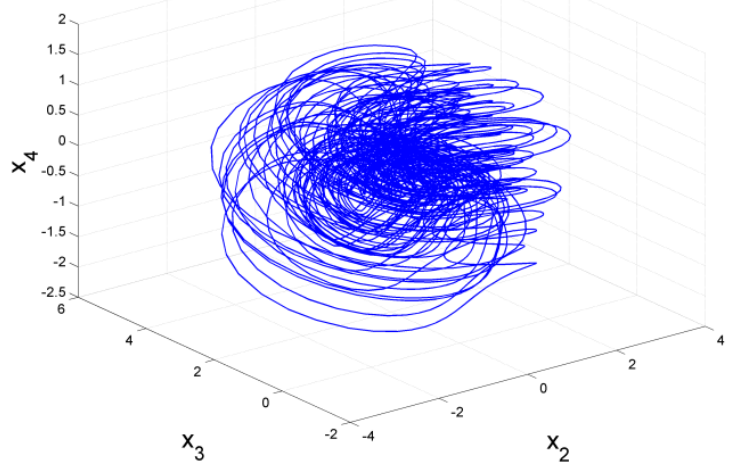

Fig. 5. The 3-D projection of the hyperchaotic Rikitake dynamo system on the $\left(x_{2}, x_{3}, x_{4}\right)$ space.

\section{Analysis of the 4-D Hyperchaotic Rikitake System}

In this section, qualitative properties of the 4-D novel hyperchaotic system are detailed.

\subsection{Dissipativity}

In vector notation, we may express the system (3) as:

$\frac{d \boldsymbol{x}}{d t}=f(\boldsymbol{x})=\left[\begin{array}{l}f_{1}\left(x_{1}, x_{2}, x_{3}, x_{4}\right) \\ f_{2}\left(x_{1}, x_{2}, x_{3}, x_{4}\right) \\ f_{3}\left(x_{1}, x_{2}, x_{3}, x_{4}\right) \\ f_{4}\left(x_{1}, x_{2}, x_{3}, x_{4}\right)\end{array}\right]$

where

$\left\{\begin{array}{l}f_{1}\left(x_{1}, x_{2}, x_{3}, x_{4}\right)=-a x_{1}+x_{2} x_{3}-p x_{4} \\ f_{2}\left(x_{1}, x_{2}, x_{3}, x_{4}\right)=-a x_{2}+x_{1}\left(x_{3}-b\right)-p x_{4} \\ f_{3}\left(x_{1}, x_{2}, x_{3}, x_{4}\right)=1-x_{1} x_{2} \\ f_{4}\left(x_{1}, x_{2}, x_{3}, x_{4}\right)=c x_{2}\end{array}\right.$

We take the parameter values as in the chaotic case (4).

Let $\Omega$ be any region in $\boldsymbol{R}^{4}$ with a smooth boundary and also $\Omega(t)=\Phi_{t}(\Omega)$, where $\Phi_{t}$ is the flow of $f$.

Furthermore, let $V(t)$ denote the hypervolume of $\Omega(t)$.

By Liouville's theorem, we have

$\frac{d V}{d t}=\int_{\Omega(t)}(\nabla \cdot f) d x_{1} d x_{2} d x_{3} d x_{4}$

The divergence of the novel hyperchaotic Rikitake dynamo system (3) is easily found as:

$\nabla \cdot f=\frac{\partial f_{1}}{\partial x_{1}}+\frac{\partial f_{2}}{\partial x_{2}}+\frac{\partial f_{3}}{\partial x_{3}}+\frac{\partial f_{4}}{\partial x_{4}}=-2 a<0$

Substituting (10) into (9), we obtain the first order ODE

$\frac{d V}{d t}=\int_{\Omega(t)}(-a) d x_{1} d x_{2} d x_{3} d x_{4}=-a V$

Integrating (11), we obtain the unique solution as:

$V(t)=\exp (-a t) V(0) \quad$ for all $t \geq 0$
From (12), we find that $V(t)$ shrinks to zero exponentially as $t \rightarrow \infty$.

Hence, the 4-D hyperchaotic Rikitake system (3) is dissipative and the asymptotic motion of the 4-D hyperchaotic system (3) settles exponentially onto a set of measure zero, i.e. a strange attractor.

\subsection{Equilibrium Points}

The equilibrium points of the novel hyperchaotic system (3) are obtained by solving the following system of equations with the parameter values as in the chaotic case (4):

$\left\{\begin{array}{lll}-a x_{1}+x_{2} x_{3}-p x_{4} & = & 0 \\ -a x_{2}+x_{1}\left(x_{3}-b\right)-p x_{4} & = & 0 \\ 1-x_{1} x_{2} & = & 0 \\ c x_{2} & = & 0\end{array}\right.$

Since the last two equations of (13) contradict each other, there is no equilibrium point for the 4-D novel hyperchaotic Rikitake system (3).

\subsection{Symmetry and Invariance}

It is easy to check that the 4-D novel hyperchaotic Rikitake system (3) is invariant under the change of coordinates

$\left(x_{1}, x_{2}, x_{3}, x_{4}\right) \mapsto\left(-x_{1},-x_{2}, x_{3},-x_{4}\right)$

Since the transformation (14) persists for all values of the system parameters, it follows that the 4-D hyperchaotic Rikitake system (3) has rotation symmetry about the $x_{3}$ axis. Hence, any non-trivial trajectory of the system (3) must have a twin trajectory.

It is also easy to check that the $x_{3}$ axis is invariant under the flow of the 4-D hyperchaotic Rikitake system (3). The invariant motion along the $x_{3}$ axis is characterized by the scalar dynamics $\dot{x}_{3}=1$, which is unstable.

\subsection{Lyapunov Exponents and Kaplan-Yorke Dimension}

For the chosen parameter values (4), the Lyapunov exponents of the novel 4-D system (3) are obtained using MATLAB as:

$\left\{\begin{array}{llc}L_{1}= & 0.09136 \\ L_{2}= & 0.02198 \\ L_{3}= & 0 \\ L_{4}= & -2.11190\end{array}\right.$

Since the spectrum of Lyapunov exponents (15) has two positive terms $L_{1}, L_{2}$, the system (3) is hyperchaotic.

We find that the sum of the Lyapunov exponents in (15) is negative, which shows that the hyperchaotic Rikitake dynamo system (3) is dissipative.

Also, the Kaplan-Yorke dimension of the novel hyperchaotic Rikitake dynamo system (3) is calculated as:

$D_{K Y}=3+\frac{L_{1}+L_{2}+L_{3}}{\left|L_{4}\right|}=3.05367$

Fig. 5 depicts the dynamics of the Lyapunov exponents of the novel hyperchaotic Rikitake system (3). 


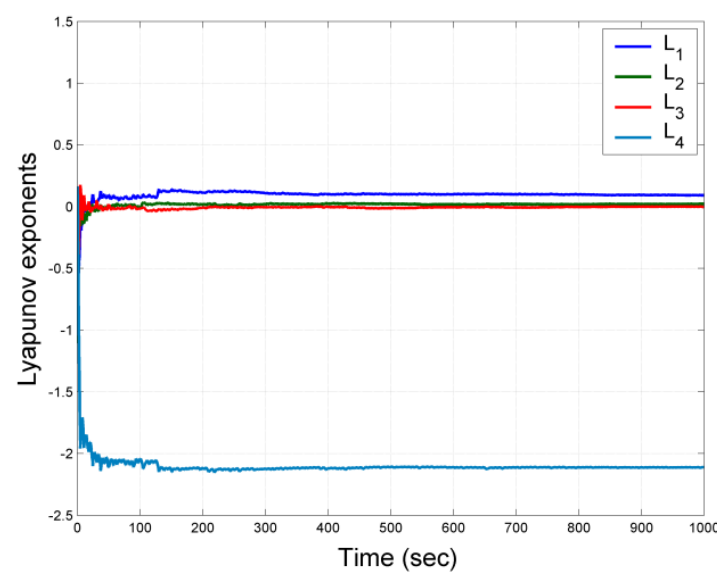

Fig. 6. The dynamics of the Lyapunov exponents of the hyperchaotic Rikitake dynamo system.

\section{Adaptive Control of the 4-D Novel Hyperchaotic Rikitake Dynamo System}

In this section, we construct an adaptive controller for globally stabilizing the 4-D novel hyperchaotic Rikitake dynamo system with unknown parameters.

We consider the controlled hyperchaotic system

$$
\left\{\begin{array}{l}
\frac{d x_{1}}{d t}=-a x_{1}+x_{2} x_{3}-p x_{4}+u_{1} \\
\frac{d x_{2}}{d t}=-a x_{2}+x_{1}\left(x_{3}-b\right)-p x_{4}+u_{2} \\
\frac{d x_{3}}{d t}=1-x_{1} x_{2}+u_{3} \\
\frac{d x_{4}}{d t}=c x_{2}+u_{4}
\end{array}\right.
$$

where $x_{1}, x_{2}, x_{3}, x_{4}$ are the state variables, $a, b, c, p$ are unknown, constant, parameters and $u_{1}, u_{2}, u_{3}, u_{4}$ are adaptive controls to be designed using estimates $\hat{a}(t), \hat{b}(t), \hat{c}(t), \hat{p}(t)$ of the unknown parameters $a, b, c, p$, respectively.

We consider the adaptive controller defined by

$$
\left\{\begin{array}{l}
u_{1}=\hat{a}(t) x_{1}-x_{2} x_{3}+\hat{p}(t) x_{4}-k_{1} x_{1} \\
u_{2}=\hat{a}(t) x_{2}-x_{1}\left(x_{3}-\hat{b}(t)\right)+\hat{p}(t) x_{4}-k_{2} x_{2} \\
u_{3}=-1+x_{1} x_{2}-k_{3} x_{3} \\
u_{4}=-\hat{c}(t) x_{2}-k_{4} x_{4}
\end{array}\right.
$$

where $k_{1}, k_{2}, k_{3}, k_{4}$ are positive gain constants, and $\hat{a}(t), \hat{b}(t), \quad \hat{c}(t), \hat{p}(t)$ are estimates of the unknown parameters $a, b, c, p$, respectively.

Substituting (18) into (17), we obtain

$$
\left\{\begin{aligned}
\frac{d x_{1}}{d t}= & -(a-\hat{a}(t)) x_{1}-(p-\hat{p}(t)) x_{4}-k_{1} x_{1} \\
\frac{d x_{2}}{d t}= & -(a-\hat{a}(t)) x_{2}-(b-\hat{b}(t)) x_{1} \\
& -(p-\hat{p}(t)) x_{4}-k_{2} x_{2} \\
\frac{d x_{3}}{d t}= & -k_{3} x_{3} \\
\frac{d x_{4}}{d t}= & (c-\hat{c}(t)) x_{2}-k_{4} x_{4}
\end{aligned}\right.
$$

The parameter estimation errors are defined by

$$
\left\{\begin{array}{l}
e_{a}(t)=a-\hat{a}(t) \\
e_{b}(t)=b-\hat{b}(t) \\
e_{c}(t)=c-\hat{c}(t) \\
e_{p}(t)=p-\hat{p}(t)
\end{array}\right.
$$

Using (20), the closed-loop state dynamics (19) can be simplified as follows:

$$
\left\{\begin{array}{l}
\frac{d x_{1}}{d t}=-e_{a} x_{1}-e_{p} x_{4}-k_{1} x_{1} \\
\frac{d x_{2}}{d t}=-e_{a} x_{2}-e_{b} x_{1}-e_{p} x_{4}-k_{2} x_{2} \\
\frac{d x_{3}}{d t}=-k_{3} x_{3} \\
\frac{d x_{4}}{d t}=e_{c} x_{2}-k_{4} x_{4}
\end{array}\right.
$$

Differentiating (22) with respect to $t$, we get

$\left\{\begin{array}{l}\frac{d e_{a}}{d t}=-\frac{d \hat{a}}{d t} \\ \frac{d e_{b}}{d t}=-\frac{d \hat{b}}{d t} \\ \frac{d e_{c}}{d t}=-\frac{d \hat{c}}{d t} \\ \frac{d e_{p}}{d t}=-\frac{d \hat{p}}{d t}\end{array}\right.$

Next, we use Lyapunov stability theory for finding an update law for the parameter estimates.

Consider the quadratic Lyapunov function defined by

$V=\frac{1}{2}\left(x_{1}^{2}+x_{2}^{2}+x_{3}^{2}+x_{4}^{2}+e_{a}^{2}+e_{b}^{2}+e_{c}^{2}+e_{p}^{2}\right)$,

which is positive definite on $R^{8}$.

Differentiating $V$ along the trajectories of (21) and (22), we get

$$
\begin{aligned}
\frac{d V}{d t}= & -k_{1} x_{1}^{2}-k_{2} x_{2}^{2}-k_{3} x_{3}^{2}-k_{4} x_{4}^{2} \\
& +e_{a}\left[-x_{1}^{2}-x_{2}^{2}-\frac{d \hat{a}}{d t}\right] \\
& +e_{b}\left[-x_{1} x_{2}-\frac{d \hat{b}}{d t}\right]+e_{c}\left[x_{2} x_{4}-\frac{d \hat{c}}{d t}\right] \\
& +e_{p}\left[-x_{4}\left(x_{1}+x_{2}\right)-\frac{d \hat{p}}{d t}\right]
\end{aligned}
$$

In view of (24), we take the parameter update law as

$\left\{\begin{array}{l}\frac{d \hat{a}}{d t}=-x_{1}^{2}-x_{2}^{2} \\ \frac{d \hat{b}}{d t}=-x_{1} x_{2} \\ \frac{d \hat{c}}{d t}=x_{2} x_{4} \\ \frac{d \hat{p}}{d t}=-x_{4}\left(x_{1}+x_{2}\right)\end{array}\right.$

Next, we state and prove the main result of this section.

Theorem 1. The novel hyperchaotic Rikitake dynamo system (17) is globally and exponentially stabilized by the adaptive control law (18) and the parameter update law (25) for all initial conditions, where $k_{1}, k_{2}, k_{3}, k_{4}$ are positive gain constants.

Proof. We prove this result using Lyapunov stability theory. For this purpose, we consider the quadratic Lyapunov 
function $V$ defined by (23), which is positive definite on $R^{8}$.

Substituting the parameter update law (25) into (24), we obtain the time derivative of $V$ as:

$\frac{d V}{d t}=-k_{1} x_{1}^{2}-k_{2} x_{2}^{2}-k_{3} x_{3}^{2}-k_{4} x_{4}^{2}$,

which is a negative semi-definite function on $R^{8}$.

Thus, we can conclude that the state vector $x(t)$ and the parameter estimation error are globally bounded.

We define $k=\min \left\{k_{1}, k_{2}, k_{3}, k_{4},\right\}$. Then we get

$\frac{d V}{d t} \leq-k\|x\|^{2}$ or $k\|x\|^{2} \leq-\frac{d V}{d t}$

Integrating the inequality (27) from 0 to $t$, we get

$k \int_{0}^{t}\|x(\tau)\|^{2} d \tau \leq V(0)-V(t)$

From (28), it follows that $x(t) \in L_{2}$. Using (21), we can conclude that $\dot{x} \in L_{\infty}$.

Thus, using Barbalat's lemma [177], we conclude that $x(t) \rightarrow 0$ exponentially as $t \rightarrow \infty$ for all initial conditions $x(0) \in R^{4}$.

This completes the proof.

For numerical simulations, the parameter values of the novel hyperchaotic Rikitake dynamo system (17) are taken as in the hyperchaotic case, viz. $a=1, b=1, c=0.7$ and $p=1.7$. We take the gain constants as $k_{i}=5$ for $i=1,2,3,4$.

The initial conditions of the hyperchaotic Rikitake dynamo system $(17)$ are taken as $x_{1}(0)=3.5, x_{2}(0)=1.7$, $x_{3}(0)=-4.5$, and $x_{4}(0)=2.8$.

The initial conditions of the parameter estimates are taken as $\hat{a}(0)=7, \hat{b}(0)=10, \hat{c}(0)=22$ and $\hat{p}(0)=18$. Figure 7 describes the time-history of the state $x(t)$.

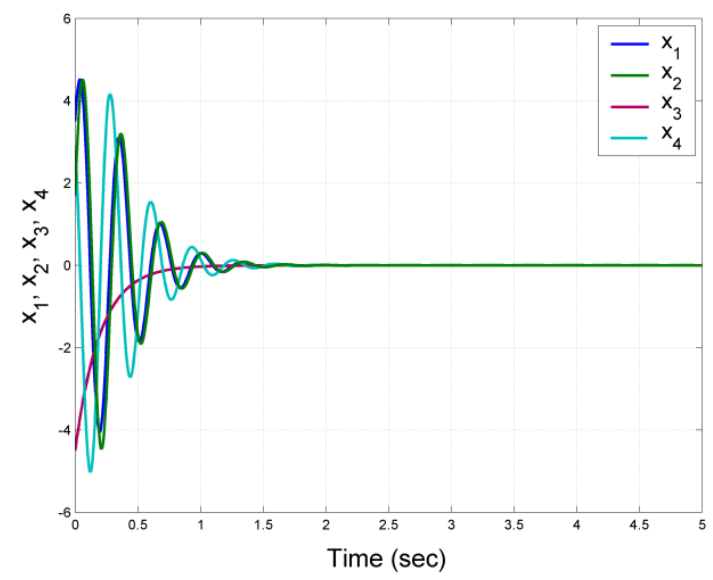

Fig. 7. Time-history of the controlled states $x_{1}, x_{2}, x_{3}, x_{4}$ of the hyperchaotic Rikitake dynamo system.

\section{Adaptive Synchronization of Identical 4-D Novel Hyperchaotic Rikitake Dynamo Systems}

In this section, we construct an adaptive synchronizer for global synchronization of identical 4-D novel hyperchaotic Rikitake dynamo systems. The adaptive synchronizer design is carried out using Lyapunov stability theory.
As the master system, we take the hyperchaotic Rikitake dynamo system

$\left\{\begin{array}{l}\frac{d x_{1}}{d t}=-a x_{1}+x_{2} x_{3}-p x_{4} \\ \frac{d x_{2}}{d t}=-a x_{2}+x_{1}\left(x_{3}-b\right)-p x_{4} \\ \frac{d x_{3}}{d t}=1-x_{1} x_{2} \\ \frac{d x_{4}}{d t}=c x_{2}\end{array}\right.$

where $x_{1}, x_{2}, x_{3}, x_{4}$ are state variables and $a, b, c, p$ are unknown, constant, parameters.

As the slave system, we take the novel hyperchaotic Rikitake dynamo system

$\left\{\begin{array}{l}\frac{d y_{1}}{d t}=-a y_{1}+y_{2} y_{3}-p y_{4}+u_{1} \\ \frac{d y_{2}}{d t}=-a y_{2}+y_{1}\left(y_{3}-b\right)-p y_{4}+u_{2} \\ \frac{d y_{3}}{d t}=1-y_{1} y_{2}+u_{3} \\ \frac{d y_{4}}{d t}=c y_{2}+u_{4}\end{array}\right.$

where $y_{1}, y_{2}, y_{3}, y_{4}$ are state variables and $u_{1}, u_{2}, u_{3}, u_{4}$ are adaptive controls.

The complete synchronization error between the systems (29) and (30) is defined as:

$\left\{\begin{array}{l}e_{1}=y_{1}-x_{1} \\ e_{2}=y_{2}-x_{2} \\ e_{3}=y_{3}-x_{3} \\ e_{4}=y_{4}-x_{4}\end{array}\right.$

The error dynamics is easily obtained as:

$\left\{\begin{array}{l}\frac{d e_{1}}{d t}=-a e_{1}-p e_{4}+y_{2} y_{3}-x_{2} x_{3}+u_{1} \\ \frac{d e_{2}}{d t}=-a e_{2}-p e_{4}-b e_{1}+y_{1} y_{3}-x_{1} x_{3}+u_{2} \\ \frac{d e_{3}}{d t}=-y_{1} y_{2}+x_{1} x_{2}+u_{3} \\ \frac{d e_{4}}{d t}=c e_{2}+u_{4}\end{array}\right.$

We consider the adaptive controller defined by

$$
\left\{\begin{aligned}
u_{1}= & \widehat{a}(t) e_{1}+\hat{p}(t) e_{4}-y_{2} y_{3}+x_{2} x_{3}-k_{1} e_{1} \\
u_{2}= & \widehat{a}(t) e_{2}+\hat{p}(t) e_{4}+\widehat{b}(t) e_{1} \\
& -y_{1} y_{3}+x_{1} x_{3}-k_{2} e_{2} \\
u_{3}= & y_{1} y_{2}-x_{1} x_{2}-k_{3} e_{3} \\
u_{4}= & -\hat{c}(t) e_{2}-k_{4} e_{4}
\end{aligned}\right.
$$

where $k_{1}, k_{2}, k_{3}, k_{4}$ are positive gain constants, and $\hat{a}(t), \hat{b}(t), \quad \hat{c}(t), \hat{p}(t)$ are estimates of the unknown parameters $a, b, c, p$, respectively.

Substituting (35) into (34), we get

$$
\left\{\begin{aligned}
\frac{d e_{1}}{d t}= & -(a-\hat{a}(t)) e_{1}-(p-\hat{p}(t)) e_{4}-k_{1} e_{1} \\
\frac{d e_{2}}{d t}= & -(a-\hat{a}(t)) e_{2}-(p-\hat{p}(t)) e_{4} \\
& -(b-\hat{b}(t)) e_{1}-k_{2} e_{2} \\
\frac{d e_{3}}{d t}= & -k_{3} e_{3} \\
\frac{d e_{4}}{d t}= & (c-\hat{c}(t)) e_{2}-k_{4} e_{4}
\end{aligned}\right.
$$


The parameter estimation errors are defined by

$$
\left\{\begin{array}{l}
e_{a}(t)=a-\hat{a}(t) \\
e_{b}(t)=b-\hat{b}(t) \\
e_{c}(t)=c-\hat{c}(t) \\
e_{p}(t)=p-\hat{p}(t)
\end{array}\right.
$$

Substituting (35) into the error dynamics (34), we get

$$
\left\{\begin{array}{l}
\frac{d e_{1}}{d t}=-e_{a} e_{1}-e_{p} e_{4}-k_{1} e_{1} \\
\frac{d e_{2}}{d t}=-e_{a} e_{2}-e_{p} e_{4}-e_{b} e_{1}-k_{2} e_{2} \\
\frac{d e_{3}}{d t}=-k_{3} e_{3} \\
\frac{d e_{4}}{d t}=e_{c} e_{2}-k_{4} e_{4}
\end{array}\right.
$$

Differentiating (35) with respect to $t$, we get

$$
\left\{\begin{array}{l}
\frac{d e_{a}}{d t}=-\frac{d \hat{a}}{d t} \\
\frac{d e_{b}}{d t}=-\frac{d \hat{b}}{d t} \\
\frac{d e_{c}}{d t}=-\frac{d \hat{c}}{d t} \\
\frac{d e_{p}}{d t}=-\frac{d \hat{p}}{d t}
\end{array}\right.
$$

Consider the quadratic Lyapunov function defined by

$$
V=\frac{1}{2}\left(e_{1}^{2}+e_{2}^{2}+e_{3}^{2}+e_{4}^{2}+e_{a}^{2}+e_{b}^{2}+e_{c}^{2}+e_{p}^{2}\right)
$$

which is positive definite on $R^{8}$.

Differentiating $V$ along the trajectories of (36) and (37), we get

$$
\begin{aligned}
\frac{d V}{d t}= & -k_{1} e_{1}^{2}-k_{2} e_{2}^{2}-k_{3} e_{3}^{2}-k_{4} e_{4}^{2} \\
& +e_{a}\left[-e_{1}^{2}-e_{2}^{2}-\frac{d \hat{a}}{d t}\right]+e_{b}\left[-e_{1} e_{2}-\frac{d \hat{b}}{d t}\right] \\
& +e_{c}\left[e_{2} e_{4}-\frac{d \hat{c}}{d t}\right]+e_{p}\left[-\left(e_{1}+e_{2}\right) e_{4}-\frac{d \hat{p}}{d t}\right]
\end{aligned}
$$

In view of (39), we take the parameter update law as:

$$
\left\{\begin{array}{l}
\frac{d \hat{a}}{d t}=-e_{1}^{2}-e_{2}^{2} \\
\frac{d \hat{b}}{d t}=-e_{1} e_{2} \\
\frac{d \hat{c}}{d t}=e_{2} e_{4} \\
\frac{d \hat{p}}{d t}=-\left(e_{1}+e_{2}\right) e_{4}
\end{array}\right.
$$

Next, we shall establish the main result of this section, viz. the adaptive synchronization of the identical hyperchaotic Rikitake dynamo systems (29) and (30). We have used Lyapunov stability theory to establish this result.

Theorem 2. The novel hyperchaotic Rikitake dynamo systems (29) and (30) are globally and exponentially synchronized by the adaptive control law (33) and the parameter update law (40) for all initial conditions, where $k_{1}, k_{2}, k_{3}, k_{4}$ are positive gain constants.

Proof. We prove this result using Lyapunov stability theory. For this purpose, we consider the quadratic Lyapunov function $V$ defined by (40), which is positive definite on $R^{8}$.
Substituting the parameter update law (40) into (39), we obtain the time derivative of $V$ as:

$\frac{d V}{d t}=-k_{1} e_{1}^{2}-k_{2} e_{2}^{2}-k_{3} e_{3}^{2}-k_{4} e_{4}^{2}$

Since $\frac{d V}{d t}$ is a negative semi-definite function on $R^{8}$, we can conclude that the anti-synchronization vector $e(t)$ and the parameter estimation error are globally bounded.

We define $k=\min \left\{k_{1}, k_{2}, k_{3}, k_{4}\right\}$.

Then we get

$\frac{d V}{d t} \leq-k\|e\|^{2}$ or $k\|e\|^{2} \leq-\frac{d V}{d t}$

Integrating the inequality (42) from 0 to $t$, we get

$$
k \int_{0}^{t}\|e(\tau)\|^{2} d \tau \leq V(0)-V(t)
$$

From (43), it follows that $e(t) \in L_{2}$. Using (36), we can conclude that $\dot{e} \in L_{\infty}$.

Thus, using Barbalat's lemma [177], we conclude that $e(t) \rightarrow 0$ exponentially as $t \rightarrow \infty$ for all initial conditions $e(0) \in R^{4}$. This completes the proof.

For numerical simulations, the parameter values of the novel hyperchaotic Rikitake dynamo systems are taken as in the hyperchaotic case, viz. $a=1, b=1, c=0.7$ and $p=1.7$.

We take the gain constants as $k_{i}=5$ for $i=1,2,3,4$.

The initial conditions of the master system (29) are taken as $x_{1}(0)=3.4, x_{2}(0)=1.8, x_{3}(0)=1.2$ and $x_{4}=-1.6$.

The initial conditions of the slave system (30) are taken as $y_{1}(0)=1.2, y_{2}(0)=6.2, y_{3}(0)=-2.7$ and $y_{4}=4.5$.

The initial conditions of the parameter estimates are taken as $\hat{a}(0)=6, \hat{b}(0)=12, \hat{c}(0)=21$ and $\hat{p}(0)=14$.

Figures 8-11 describe the complete synchronization of the states of the novel hyperchaotic Rikitake dynamo systems (29) and (30).

Figure 12 describes the time-history of the synchronization error $e(t)$.

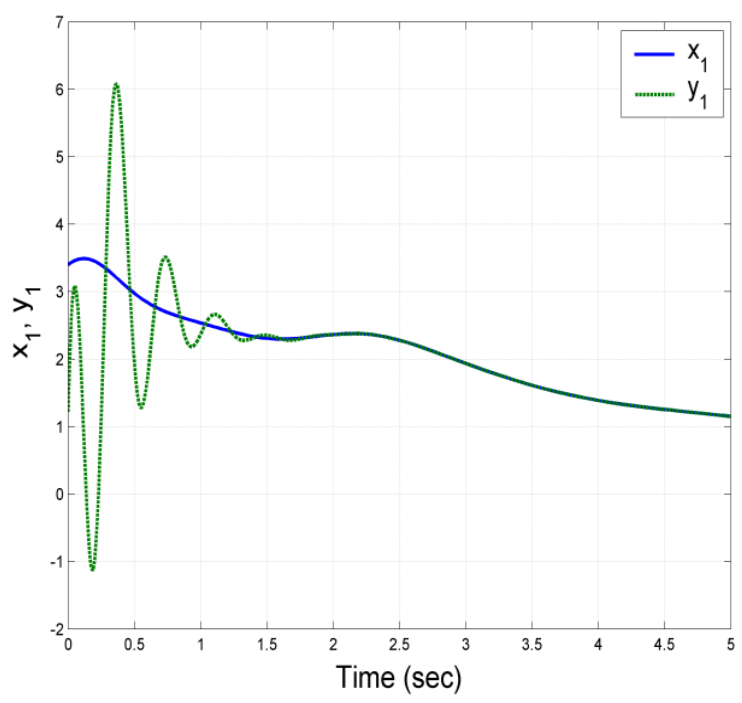

Fig. 8. The synchronization of the states $x_{1}$ and $y_{1}$. 


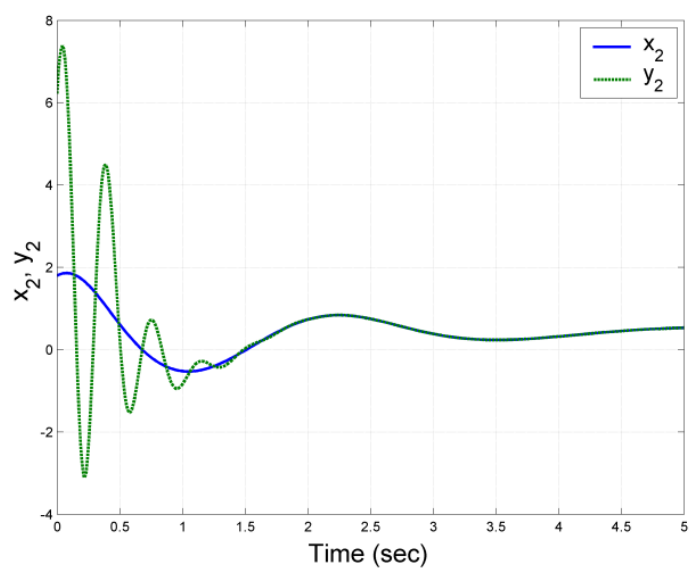

Fig. 9. The synchronization of the states $x_{2}$ and $y_{2}$.

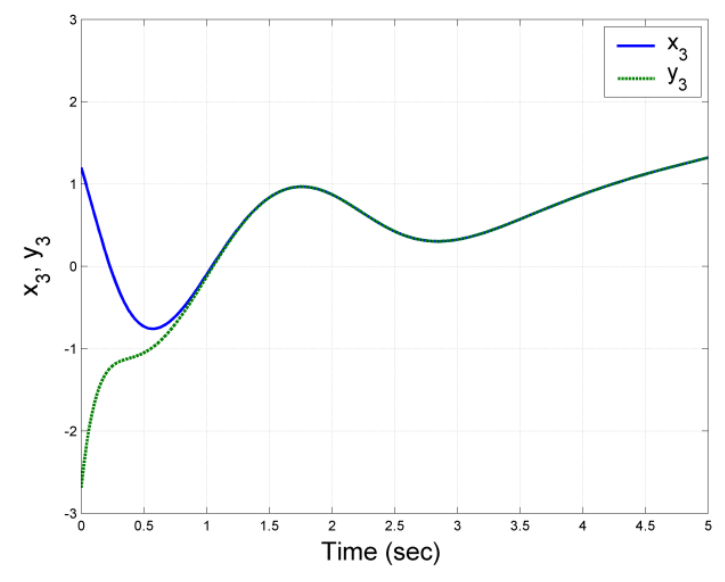

Fig. 10. The synchronization of the states $x_{3}$ and $y_{3}$.

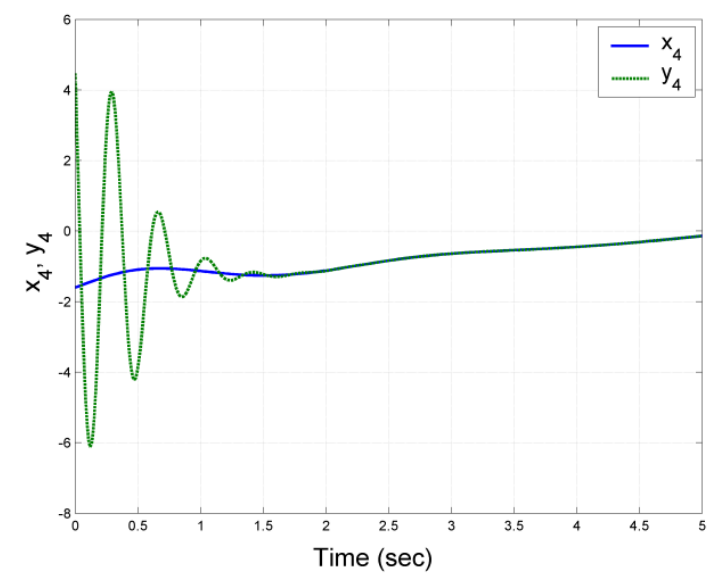

Fig. 11. The synchronization of the states $x_{4}$ and $y_{4}$.

\section{Circuit Simulation}

In this Section, an electronic circuit is constructed to implement hyperchaotic system without equilibrium (3). The circuit in Fig. 13 has been designed using a general approach based on operational amplifiers $[30,35,36]$.

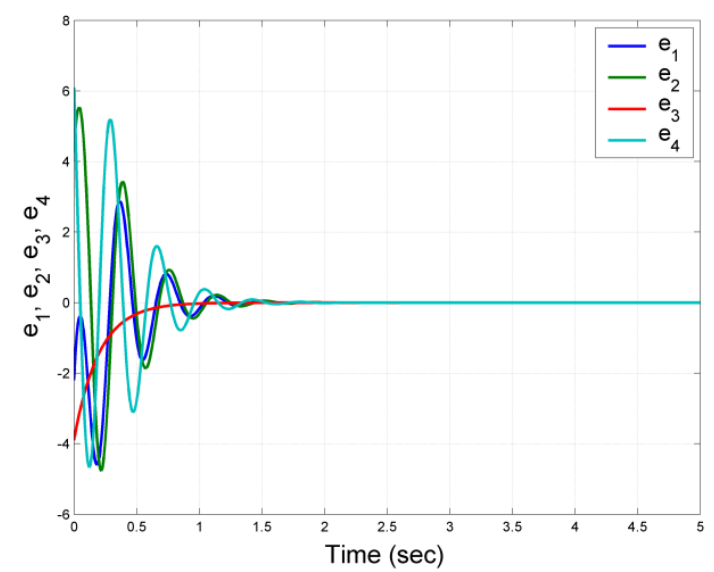

Fig. 12. Time-history of the synchronization error.
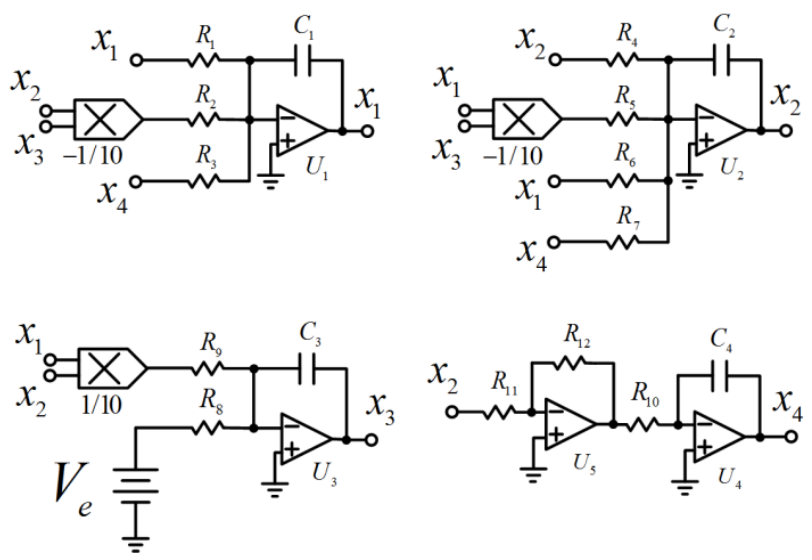

Fig. 13. Schematic of the circuit realizing the novel 4-D hyperchaotic Rikitake dynamo model (3).

By applying Kirchhoff's laws to the electronic circuit in Fig. 13, its nonlinear equations are given as

$$
\left\{\begin{array}{l}
\frac{d v_{C_{1}}}{d t}=-\frac{1}{R_{1} C_{1}} v_{C_{1}}+\frac{1}{10 R_{2} C_{1}} v_{C_{2}} v_{C_{3}}-\frac{1}{R_{3} C_{1}} v_{C_{4}} \\
\frac{d v_{C_{2}}}{d t}=-\frac{1}{R_{4} C_{2}} v_{C_{2}}+\frac{1}{10 R_{5} C_{2}} v_{C_{1}} v_{C_{3}}-\frac{1}{R_{6} C_{2}} v_{C_{1}}-\frac{1}{R_{7} C_{2}} v_{C_{4}} \\
\frac{d v_{C_{3}}}{d t}=\frac{1}{R_{8} C_{3}} V_{e}-\frac{1}{10 R_{9} C_{3}} v_{C_{1}} v_{C_{2}} \\
\frac{d v_{C_{4}}}{d t}=\frac{R_{12}}{R_{10} R_{11} C_{4}} v_{C_{2}}
\end{array}\right.
$$

where $v_{C_{1}}, v_{C_{2}}, v_{C_{3}}, v_{C_{4}}$ are the voltages across the capacitors $C_{1}, C_{2}, C_{3}$ and $C_{4}$, respectively. It is noting that the state variables $x_{1}, x_{2}, x_{3}, x_{4}$ of 4 -D hyperchaotic Rikitake dynamo model (3) are the voltages $v_{C_{1}}, v_{C_{2}}, v_{C_{3}}$, $v_{C_{4}}$ respectively.

The values of the electronic components in Fig. 13 are chosen to match parameters of 4-D hyperchaotic Rikitake dynamo model (3) as follows: $R_{1}=R_{4}=R_{6}=R_{8}=R_{11}=R_{12}=$ $10 \mathrm{k} \Omega, R_{2}=R_{5}=R_{9}=1 \mathrm{k} \Omega, R_{3}=R_{7}=5.882 \mathrm{k} \Omega, R_{10}=$ $14.286 \mathrm{k} \Omega, C_{1}=C_{2}=C_{3}=C_{4}=10 \mathrm{nF}$, and $V_{\mathrm{e}}=-1 \mathrm{~V}_{\mathrm{DC}}$. The power supplies of all active devices are $\pm 15 \mathrm{Volts}$. 
The designed circuit is implemented by using the electronic simulation package Cadence OrCAD and obtained phase portraits are presented in Figs. 14-16.

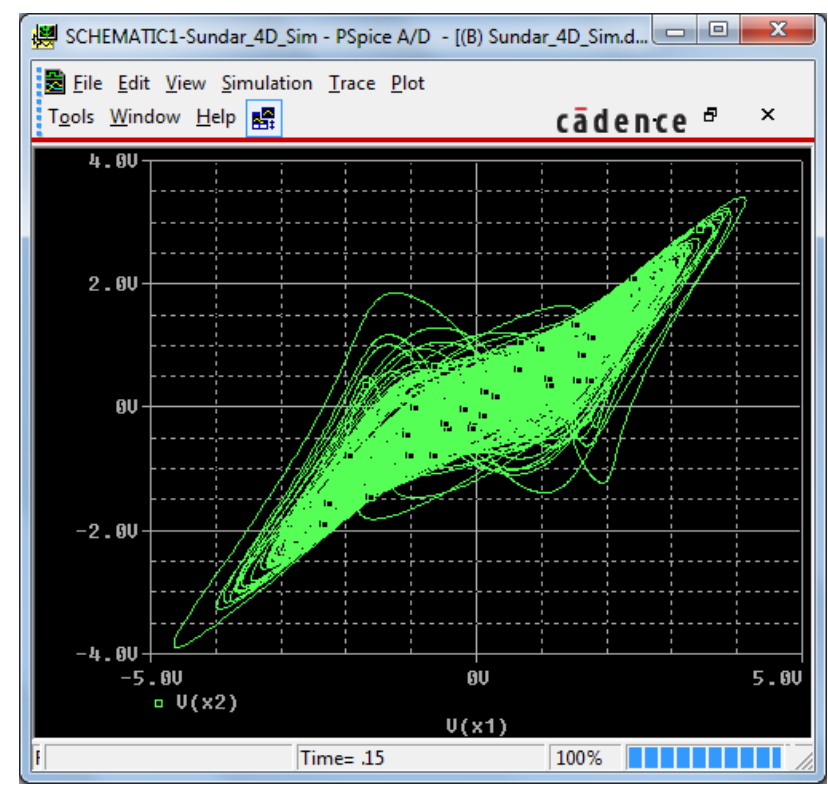

Fig. 14. Hyperchaotic attractor obtained from the proposed circuit in $\left(v_{C_{1}}, v_{C_{2}}\right)$-plane

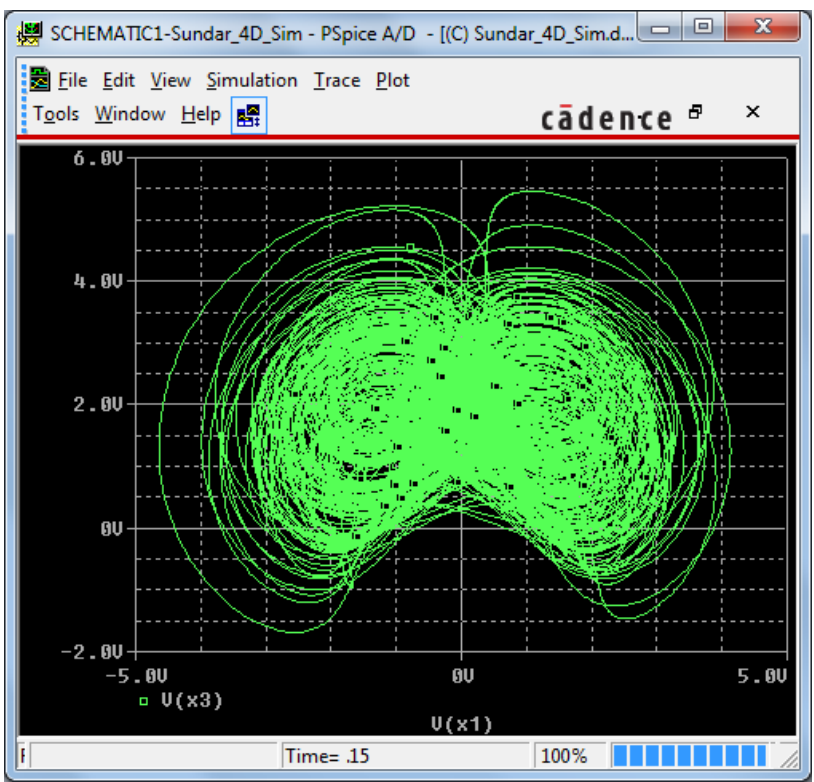

Fig. 15. Hyperchaotic attractor obtained from the proposed circuit in $\left(v_{C_{1}}, v_{C_{3}}\right)$-plane

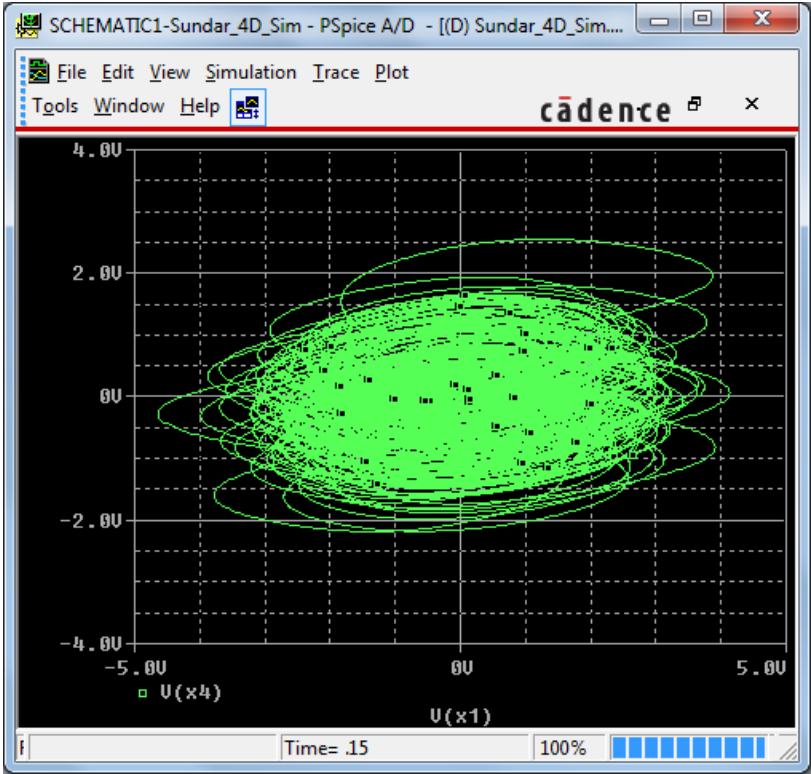

Fig. 16. Hyperchaotic attractor obtained from the proposed circuit in $\left(v_{C_{1}}, v_{C_{4}}\right)$-plane

\section{Conclusion}

In this paper, a new 4-D Rikitake dynamo model has been investigated. It is interesting that such Rikitake dynamo model can exhibit hyperchaos although it possesses no equilibrium points. As the result, this system can be classified as a hyperchaotic system with hidden strange attractor. Qualitative properties of the 4-D novel hyperchaotic system are studied. In addition, an adaptive controller and an adaptive synchronizer for such system have been proposed. Moreover, an electronic circuit modeling this new hyperchaotic system has been designed using off-the-shelf components to confirm the feasibility of theoretical model.

Recently, discovering systems with hidden attractors has come a new attractive research topic because of their practical and theoretical importance. Especially there are only few works reporting hyperchaotic hidden strange attractor. Hence this Rikitake dynamo model should be further studied in future works.

\section{References}

1. S.H. Strogatz, Nonlinear dynamics and chaos: With applications to physics, biology, chemistry, and engineering, Perseus Books, Massachusetts, US (1994)

2. E.N. Lorenz, Deterministic nonperiodic flow, Journal of the Atmospheric Sciences, vol. 20, pp. 130-141 (1963).

3. O.E. Rössler, An equation for continuous chaos, Physics Letters A, vol. 57, pp. 397-398 (1976)

4. M.I. Rabinovich and A.L. Fabrikant, Stochastic self-modulation of waves in nonequilibrium media, Sov. Phys. JETP, vol. 50, pp. 311-317, (1979)

5. A. Arneodo, P. Coullet, and C. Tresser, Possible new strange attractors with spiral structure, Communications in Mathematical Physics, vol. 79, pp. 573-579 (1981).
6. J.C. Sprott, Some simple chaotic flows, Physical Review E, vol. 50, pp. 647-650 (1994).

7. G. Chen and T. Ueta, Yet another chaotic oscillator, International Journal of Bifurcation and Chaos, vol. 9, pp. 14651466 (1999).

8. J. Lü and G. Chen, A new chaotic attractor coined, International Journal of Bifurcation and Chaos, vol. 12, pp. 659-661 (2002).

9. R. Shaw, Strange attractors, chaotic behaviour and information flow, Zeitschrift für Naturforschung, vol. 36, pp. 80-112 (1981).

10. B. Feeny and F.C. Moon, Chaos in a forced dry-friction oscillator: Experiments and numerical modeling, Journal of Sound and Vibration, vol. 170, pp. 303-323 (1994). 
11. T. Shimizu and N. Moroika, On the bifurcation of a symmetric limit cycle to an asymmetric one in a simple model, Physics Letters A, vol. 76, pp. 201-204 (1980).

12. W. Liu and G. Chen, A new chaotic system and its generation, International Journal of Bifurcation and Chaos, vol. 13, pp. 261267 (2003).

13. G. Cai and Z. Tan, Chaos synchronization of a new chaotic system via nonlinear control, Journal of Uncertain Systems, vol. 1, pp. 235-240 (2007).

14. G. Tigan and D. Opris, Analysis of a 3D chaotic system, Chaos, Solitons and Fractals, vol. 36, pp. 1315-1319 (2008).

15. G.P. Kennedy, Chaos in the Colpitts oscillator, IEEE Transactions on Circuits and Systems-I, vol. 41, pp. 771-774 (1994).

16. J. Wang, D. Lu and L. Tian, Global synchronization for time delay of WINDMI system, Chaos, Solitons and Fractals, vol. 30, pp. 629-635 (2006).

17. W. Zhou, Y. Xu, H. Lu, and L. Pan, On dynamics analysis of a new chaotic attractor, Physics Letters A, vol. 372, pp. 57735777 (2008).

18. D. Li, A three-scroll chaotic attractor, Physics Letters A, vol. 372, pp. 387-393 (2008).

19. Z. Elhadj, Dynamical analysis of a 3-D chaotic system with only two quadratic nonlinearities, Journal of Systems Science and Complexity, vol. 21, pp. 67-75 (2008).

20. L. Pan, D. Xu and W. Zhou, Controlling a novel chaotic attractor using linear feedback, Journal of Information and Computing Science, vol. 5, pp. 117-124 (2010).

21. V. Sundarapandian, Analysis and anti-synchronization of a novel chaotic system via active and adaptive controllers, Journal of Engineering Science and Technology Review, vol. 6, pp. 45-52 (2013).

22. F. Yu, C. Wang, Q. Wan, and Y. Hu, Complete switched modified function projective synchronization of a five-term chaotic system with uncertain parameters and disturbances, Pramana, vol. 80, pp. 223-235 (2013).

23. V. Sundarapandian and I. Pehlivan, Analysis, control, synchronization and circuit design of a novel chaotic system, Mathematical and Computer Modelling, vol. 55, pp. 1904-1915 (2012).

24. C. Zhu, Y. Liu, and Y. Guo, Theoretical and numerical study of a new chaotic system, Intelligent Information Management, vol. 2, pp. 104-109 (2010)

25. S. Vaidyanathan, A new six-term 3-D chaotic system with an exponential nonlinearity, Far East Journal of Mathematical Sciences, vol. 79, pp. 135-143 (2013).

26. S. Vaidyanathan, Analysis and adaptive synchronization of two novel chaotic systems with hyperbolic sinusoidal and cosinusoidal nonlinearity and unknown parameters, Journal of Engineering Science and Technology Review, vol. 6, pp. 53-65 (2013).

27. S. Vaidyanathan, A new eight-term 3-D polynomial chaotic system with three quadratic nonlinearities, Far East Journal of Mathematical Sciences, vol. 84, pp. 219-226 (2014).

28. S. Vaidyanathan, Analysis, control and synchronization of a sixterm novel chaotic system with three quadratic nonlinearities, International Journal of Modelling, Identification and Control, vol. 22, pp. 41-53 (2014).

29. S. Vaidyanathan, Analysis and adaptive synchronization of eight-term 3-D polynomial chaotic systems with three quadratic nonlinearities, European Physical Journal: Special Topics, vol. 223, pp. 1519-1529 (2014).

30. S. Vaidyanathan, Ch. Volos, V.T. Pham, K. Madhavan, and B.A. Idowu, Adaptive backstepping control, synchronization and circuit simulation of a 3-D novel jerk chaotic system with two hyperbolic sinusoidal nonlinearities, Archives of Control Sciences, vol. 24(3), pp. 257-285 (2014).

31. S. Vaidyanathan, Qualitative analysis, adaptive control and synchronization of a seven-term novel 3-D chaotic system with a quartic nonlinearity, International Journal of Control Theory and Applications, vol. 7 (1), pp. 1-20 (2014)

32. S. Vaidyanathan, Ch. K. Volos and V.T. Pham, Global chaos control of a novel nine-term chaotic system via sliding mode control, Studies in Computational Intelligence, vol. 576, pp. 571590, (2015).

33. S. Vaidyanathan and A.T. Azar, Analysis, control and synchronization of a nine-term 3-D novel chaotic system, Studies in Computational Intelligence, vol. 581, pp. 59-72, 2015.
34. S. Vaidyanathan and K. Madhavan, Analysis, adaptive control and synchronization of a seven-term novel 3-D chaotic system, International Journal of Control Theory and Applications, vol. 6, pp. 121-137 (2013).

35. I. Pehlivan, I.M. Moroz, and S. Vaidyanathan, Analysis, synchronization and circuit design of a novel butterfly attractor, Journal of Sound and Vibration, vol. 333, pp. 5077-5096 (2014).

36. V.T. Pham, Ch. K. Volos, S. Jafari, Z. Wei and X. Wang, Constructing a novel no-equilibrium chaotic system, International Journal of Bifurcation and Chaos, vol. 24, 1450073 (2014).

37. V.T. Pham, Ch. K. Volos and S. Vaidyanathan, Multi-scroll chaotic oscillator based on a first-order delay differential equation, Studies in Computational Intelligence, vol. 581, pp. 59-72 (2015).

38. Z. Wang and K.T. Chau, Control of chaotic vibration in automotive wiper systems, Chaos, Solitons and Fractals, vol. 39, no. 1, pp. 168-181 (2009).

39. J. Shi, F. Zhao, X. Shen and X. Wang, Chaotic operation and chaos control of travelling wave ultrasonic motor, Ultrasonics, vol. 53, no. 6, pp. 1112-1123 (2013).

40. D. Yang and J. Zhou, Connections among several chaos feedback control approaches and chaotic vibration control of mechanical systems, Communications in Nonlinear Science and Numerical Simulation, vol. 19, no. 11, pp. 3954-3968 (2014).

41. H.B. Fotsin and J. Daafouz, Adaptive synchronization of uncertain Colpitts oscillators based on parameter identification, Physics Letters A, vol. 339, pp. 304-315 (2005).

42. G.H. Li, S.P. Zhou and K. Yang, Controlling chaos in Colpitts oscillator, Chaos, Solitons and Fractals, vol. 33, pp. 582-587 (2007).

43. A. Sharma, V. Patidar, G. Purohit, and K.K. Sud, Effects on the bifurcation and chaos in forced Duffing oscillator due to nonlinear damping, Communications in Nonlinear Science and Numerical Simulation, vol. 17, pp. 2254-2269 (2012).

44. U. Nehmzow and K. Walker, Quantitative description of robotenvironment interaction using chaos theory, Robotics and Autonomous Systems, vol. 53, pp. 177-193 (2005).

45. S. Iqbal, X. Zang, Y. Zhu, and J. Zhao, Bifurcations and chaos in passive dynamic walking: A review, Robotics and Autonomous Systems, vol. 62, pp. 889-909 (2014).

46. Ch.K. Volos, I.M. Kyprianidis, and I.N. Stouboulos, A chaotic path planning generator for autonomous mobile robots, Robotics and Autonomous Systems, vol. 60, pp. 651-656 (2012).

47. Ch.K. Volos, I.M. Kyprianidis, and I.N. Stouboulos, Experimental investigation on coverage performance of a chaotic autonomous mobile robot, Robotics and Autonomous Systems, vol. 61(12), pp. 1314-1322 (2013).

48. J.C. Roux, Chaos in experimental chemical systems: two examples, North-Holland Mathematics Studies, vol. 103, pp. 345-352 (1985).

49. Y.N. Li, L. Chen, Z.S. Cai, and X.Z. Zhao, Study on chaos synchronization in the Belousov-Zhabotinsky chemical system, Chaos, Solitons and Fractals, vol. 17, pp. 699-707 (2003).

50. Y. Gong, Y. Xie, X. Lin, Y. Hao, and X. Ma, Ordering chaos and synchronization transitions by chemical delay and coupling on scale-free neuronal networks, Chaos, Solitons and Fractals, vol. 43, pp. 96-103 (2010).

51. M. Kyriazis, Applications of chaos theory to the molecular biology of aging, Experimental Gerontology, vol. 26, pp. 569572 (1991).

52. G. Böhm, Protein folding and deterministic chaos: Limits of protein folding simulations and calculations, Chaos, Solitons and Fractals, vol. 1, pp. 375-382 (1991).

53. J.C. Sprott, J.A. Vano, J.C. Wildenberg, M.B. Anderson, and J.K. Noel, Coexistence and chaos in complex ecologies, Physics Letters A, vol. 335, pp. 207-212 (2005).

54. B. Sahoo and S. Poria, The chaos and control of a food chain model supplying additional food to top-predator, Chaos, Solitons and Fractals, vol. 58, pp. 52-64 (2014).

55. T.A. Denton, G.A. Diamond, R.H. Helfant, S. Khan, and H. Karagueuzian, Fascinating rhythm: A primer on chaos theory and its applications to cardiology, American Heart Journal, vol. 120, pp. 1419-1440 (1990).

56. A. Wu, S. Wen, and Z. Zeng, Synchronization control of a class of memristor-based neural networks, Information Sciences, vol. 183, pp. 106-116, (2012). 
57. S. Wen, Z. Zeng, and T. Huang, Adaptive synchronization of memristor-based Chua's circuits, Physics Letters A, vol. 376, pp. 2775-2780, (2012).

58. A. Wu, and Z. Zeng, Anti-synchronization control of a class of memristive recurrent neural networks, Communications in Nonlinear Science and Numerical Simulation, vol. 18, pp. 373385 (2013).

59. G. He, Z. Cao, P. Zhu, and H. Ogura, Controlling chaos in a chaotic neural network, Neural Networks, vol. 16, pp. 11951200 (2003).

60. E. Kaslik and S. Sivasundaram, Nonlinear dynamics and chaos in fractional-order neural networks, Neural Networks, vol. 32, pp. 245-256 (2012).

61. I.M. Kyprianidis and A.T. Makri, Complex dynamics of FitzHugh-Nagumo type neurons coupled with gap junction under external voltage stimulation, Journal of Engineering Science and Technology Review, vol. 6(4), pp. 104-114 (2013).

62. K. Suzuki and Y. Imai, Decryption characteristics in message modulation type chaos secure communication system using optical fiber ring resonators, Optics Communications, vol. 259, pp. 88-93 (2006).

63. X.Y. Wang and Y.F. Gao, A switch-modulated method for chaos digital secure communication based on user-defined protocol, Communications in Nonlinear Science and Numerical Simulation, vol. 15, pp. 99-104 (2010).

64. O.I. Moskalenko, A.A. Koronovskii, and A.E. Hramov, Generalized synchronization of chaos for secure communication: Remarkable stability to noise, Physics Letters A, vol. 374, pp. 2925-2931 (2010)

65. A. Abdullah, Synchronization and secure communication of uncertain chaotic systems based on full-order and reduced-order output-affine observers, Applied Mathematics and Computation, vol. 219, pp. 10000-10011 (2013).

66. N. Smaoui and A. Kanso, Cryptography with chaos and shadowing, Chaos, Solitons and Fractals, vol. 42, pp. 2312-2321 (2009).

67. R. Rhouma and S. Belghith, Cryptanalysis of a chaos-based cryptosystem on DSP, Communications in Nonlinear Science and Numerical Simulation, vol. 16, pp. 876-884 (2011).

68. Ch K. Volos, I.M. Kyprianidis, and I.N. Stouboulos, Text encryption scheme realized with a chaotic pseudo-random bit generator, Journal of Engineering Science and Technology Review, vol. 6(4), pp. 9-14 (2013)

69. Ch.K. Volos, I.M. Kyprianidis, and I.N. Stouboulos, Image encryption process based on chaotic synchronization phenomena, Signal Processing, vol. 93(5), pp. 1328-1340 (2013).

70. J. Yang, D. Xiao and T. Xiang, Cryptanalysis of a chaos block cipher for wireless sensor network, Communications in Nonlinear Analysis and Numerical Simulation, vol. 16(2), pp. 844-850 (2011)

71. N. Bezzo, P.J. Cruz, F. Sorrentino and R. Fierro, Decentralized identification and control of networks of coupled mobile platforms through adaptive synchronization of chaos, Physica D: Nonlinear Phenomena, vol. 267, pp. 94-103 (2014)

72. D. Guégan, Chaos in economics and finance, Annual Reviews in Control, vol. 33, pp. 89-93 (2009).

73. Ch.K. Volos, I.M. Kyprianidis, and I.N. Stouboulos, Synchronization phenomena in coupled nonlinear systems applied in economic cycles, WSEAS Trans. Systems, vol. 11(12), pp. 681-690 (2012).

74. P. Caraiani, Testing for nonlinearity and chaos in economic time series with noise titration, Economics Letters, vol. 120, pp. 192194 (2013)

75. A. Kazem, E. Sharifi, F.K. Hussain, M. Saberi and O.K. Hussain, Support vector regression with chaos-based firefly algorithm for stock market price forecasting, Applied Soft Computing, vol. 13(2), pp. 947-958 (2013).

76. V. Sundarapandian, Output regulation of the Lorenz attractor, Far East Journal of Mathematical Sciences, vol. 42, pp. 289-299 (2010).

77. C.A. Kitio Kwuimy and B.R. Nana Nbendjo, Active control of horseshoes chaos in a driven Rayleigh oscillator with fractional order deflection, Physics Letters A, vol. 375 (39), pp. 3442-3449 (2011).

78. S. Vaidyanathan, Output regulation of Arneodo-Coullet chaotic system, Communications in Computer and Information Science, vol. 131, pp. 585-593 (2011).
79. S. Vaidyanathan, Output regulation of the unified chaotic system, Communications in Computer and Information Science, vol. 198, pp. 1-9 (2011).

80. S. Vaidyanathan, Output regulation of the Liu chaotic system, Applied Mechanics and Materials, vols. 110-116, pp. 3982-3989 (2012).

81. G. Chen, A simple adaptive feedback control method for chaos and hyper-chaos control, Applied Mathematics and Computation, vol. 217, pp. 7258-7264 (2011).

82. J. Zheng, A simple universal adaptive feedback controller for chaos and hyperchaos control, Computers \& Mathematics with Applications, vol. 61, pp. 2000-2004 (2011).

83. S. Vaidyanathan, Adaptive controller and synchronizer design for the Qi-Chen chaotic system, Lecture Notes of the Institute for Computer Sciences, Social-Informatics and Telecommunications Engineering, vol. 85, pp. 124-133 (2012).

84. V. Sundarapandian, Adaptive control and synchronization design for the Lu-Xiao chaotic system, Lecture Notes in Electrical Engineering, vol. 131, pp. 319-327 (2013).

85. S. Vaidyanathan, A ten-term novel 4-D hyperchaotic system with three quadratic nonlinearities and its control, International Journal of Control Theory and Applications, vol. 6, pp. 97-109 (2013).

86. S. Vaidyanathan, Analysis, control and synchronization of hyperchaotic Zhou system via adaptive control, Advances in Intelligent Systems and Computing, vol. 177, pp. 1-10 (2013).

87. D. Yang and J. Zhou, Connections among several chaos feedback control approaches and chaotic vibration control of mechanical systems, Communications in Nonlinear Science and Numerical Simulation, vol. 19, pp. 3954-3968 (2014).

88. M.T. Yassen, Chaos control of chaotic dynamical systems using backstepping design, Chaos, Solitons and Fractals, vol. 27, pp. 537-548 (2006).

89. J.A. Laoye, U.E. Vincent, and S.O. Kareem, Chaos control of 4D chaotic systems using recursive backstepping nonlinear controller, Chaos, Solitons and Fractals, vol. 39, pp. 356-362 (2009).

90. D. Lin, X. Wang, F. Nian, and Y. Zhang, Dynamic fuzzy neural networks modeling and adaptive backstepping tracking control of uncertain chaotic systems, Neurocomputing, vol. 73, pp. 2873-2881 (2010).

91. S. Vaidyanathan, Sliding mode control based global chaos control of Liu-Liu-Liu-Su chaotic system, International Journal of Control Theory and Applications, vol. 5, pp. 15-20 (2012).

92. S. Vaidyanathan, Global chaos control of hyperchaotic Liu system via sliding mode control, vol. 5, pp. 117-123 (2012).

93. C. Liu, X. Liao and K.W. Wong, Lag synchronization of hyperchaos with application to secure communication, Chaos, Solitons and Fractals, vol. 23, pp. 183-193 (2005).

94. N. Smaoui, A. Karouma and M. Zribi, Secure communications based on the synchronization of the hyperchaotic Chen and the unified chaotic systems, Communications in Nonlinear Science and Numerical Simulation, vol. 16, pp. 3279-3293 (2011).

95. X.J. Wu, H. Wang and H.T. Lu, Hyperchaotic secure communication via generalized function projective synchronization, Nonlinear Analysis: Real World Applications, vol. 12 , pp. 1288-1299 (2011).

96. T.I. Chien and T.L. Liao, Design of secure digital communication systems using chaotic modulation, cryptography and chaotic synchronization, Chaos, Solitons and Fractals, vol. 24, pp. 241-245 (2005).

97. B. Zhang, M. Chen and D. Zhou, Chaotic secure communication based on particle filtering, Chaos, Solitons and Fractals, vol. 30, pp. 1273-1280 (2006).

98. X. Wu, C. Bai and H. Kan, A new color image cryptosystem via hyperchaos synchronization, Communications in Nonlinear Science and Numerical Simulation, vol. 19, pp. 1884-1897 (2014).

99. Q. Zhang, L. Guo and X. Wei, A novel image fusion encryption algorithm based on DNA sequence operation and hyper-chaotic system, Optik-International Journal for Light and Electron Optics, vol. 124, pp. 3596-3600 (2013).

100. G. Ye and J. Zhou, A block chaotic image encryption scheme based on self-adaptive modelling, Applied Soft Computing, vol. 22, pp. 351-357, (2014).

101. H. Liu, X. Wang and A. Kadir, Color image encryption using Choquet fuzzy integral and hyper chaotic system, Optik- 
International Journal for Light and Electron Optics, vol. 124, pp. 3257-3533 (2013).

102. A. Buscarino, L. Fortuna and M. Frasca, Experimental robust synchronization of hyperchaotic circuits, Physica D: Nonlinear Phenomena, vol. 238, pp. 1917-1922, (2009).

103. N. Yujun, W. Xingyuan, W. Mingjun and Z. Huaguang, A new hyperchaotic system and its circuit implementation, Communications in Nonlinear Science and Numerical Simulation, vol. 15, pp. 3518-3524 (2010).

104. X. Wei, F. Yunfei and L. Qiang, A novel four-wing hyperchaotic system and its circuit implementation, Procedia Engineering, vol. 29, pp. 1264-1269 (2012).

105. P. Zhou and K. Huang, A new 4-D non-equilibrium fractionalorder chaotic system and its circuit implementation, Communications in Nonlinear Science and Numerical Simulation, vol. 19, pp. 2005-2011 (2014).

106. L. Kocarev and U. Parlitz, General approach for chaos synchronization with applications to communications, Physical Review Letters, vol. 74, pp. 5028-5030 (1995).

107. K. Murali and M. Lakshmanan, Secure communication using a compound signal using sampled-data feedback, Applied Mathematics and Mechanics, vol. 11, pp. 1309-1315 (1995).

108. M. Feki, An adaptive chaos synchronization scheme applied to secure communication, Chaos, Solitons and Fractals, vol. 18, pp. 141-148 (2003).

109. J. Yang and F. Zhu, Synchronization for chaotic systems and chaos-based secure communications via both reduced-order and step-by-step sliding mode observers, Communications in Nonlinear Science and Numerical Simulation, vol. 18, pp. 926937 (2013).

110. L. Kocarev, Chaos-based cryptography: a brief overview, IEEE Circuits and Systems, vol. 1, pp. 6-21 (2001).

111. H. Gao, Y. Zhang, S. Liang and D. Li, A new chaotic algorithm for image encryption, Chaos, Solitons and Fractals, vol. 29, pp. 393-399 (2006).

112. Y. Wang, K.W. Wang, X. Liao and G. Chen, A new chaos-based fast image encryption, Applied Soft Computing, vol. 11, pp. 514-522 (2011)

113. Y. Xu, H. Wang, Y. Li and B. Pei, Image encryption based on synchronization of fractional chaotic systems, Communications in Nonlinear Science and Numerical Simulation, vol. 19(10), pp. 3735-3744 (2014).

114. L.M. Pecora and T.L. Carroll, Synchronization in chaotic systems, vol. 64, pp. 821-825 (1990).

115. S. Vaidyanathan and S. Rasappan, New results on the global chaos synchronization for Liu-Chen-Liu and Lü chaotic systems, Communications in Computer and Information Science, vol. 102, pp. 20-27 (2010).

116. S. Vaidyanathan and S. Rasappan, Hybrid synchronization of hyperchaotic Qi and Lü systems by nonlinear control, Communications in Computer and Information Science, vol. 131, pp. 585-593 (2011).

117. S. Vaidyanathan and K. Rajagopal, Anti-synchronization of Li and $\mathrm{T}$ chaotic systems by active nonlinear control, Communications in Computer and Information Science, vol. 198, pp. 175-184 (2011).

118. S. Vaidyanathan and S. Rasappan, Global chaos synchronization of hyperchaotic Bao and Xu systems by active nonlinear control, Communications in Computer and Information Science, vol. 198, pp. 10-17 (2011).

119. S. Vaidyanathan and K. Rajagopal, Global chaos synchronization of hyperchaotic Pang and Wang systems by active nonlinear control, Communications in Computer and Information Science, vol. 204, pp. $84-93$ (2011).

120. S. Vaidyanathan, Hybrid chaos synchronization of Liu and Lü systems by active nonlinear control, Communications in Computer and Information Science, vol. 204, pp. 1-10 (2011).

121. P. Sarasu and V. Sundarapandian, Active controller design for generalized projective synchronization of four-scroll chaotic systems, International Journal of Systems Signal Control and Engineering Application, vol. 4, pp. 26-33 (2011).

122. S. Vaidyanathan and K. Rajagopal, Hybrid synchronization of hyperchaotic Wang-Chen and hyperchaotic Lorenz systems by active non-linear control, International Journal of Systems Signal Control and Engineering Application, vol. 4, pp. 55-61 (2011).

123. S. Pakiriswamy and S. Vaidyanathan, Generalized projective synchronization of three-scroll chaotic systems via active control, Lecture Notes of the Institute for Computer Sciences, Social-Informatics and Telecommunications Engineering, vol. 85, pp. 146-155 (2012).

124. V. Sundarapandian and R. Karthikeyan, Hybrid synchronization of hyperchaotic Lorenz and hyperchaotic Chen systems via active control, Journal of Engineering and Applied Sciences, vol. 7, pp. 254-264 (2012).

125. R. Karthikeyan and V. Sundarapandian, Hybrid chaos synchronization of four-scroll systems via active control, Journal of Electrical Engineering, vol. 65, pp. 97-103 (2014).

126. E.M. Shahverdiev and K.A. Shore, Impact of modulated multiple optical feedback time delays on laser diode chaos synchronization, Optics Communications, vol. 282, pp. 35683572 (2009).

127. T. Botmart, P. Niamsup, and X. Liu, Synchronization of nonautonomous chaotic systems with time-varying delay via delayed feedback control, Communications in Nonlinear Science and Numerical Simulation, vol. 17, pp. 1894-1907 (2012).

128. S. Bowong, Adaptive synchronization between two different chaotic dynamical systems, Communications in Nonlinear Science and Numerical Simulation, vol. 12, pp. 976-985 (2007).

129. H. Salarieh and A. Alasty, Adaptive chaos synchronization in Chua's systems with noisy parameters, Mathematics and Computers in Simulation, vol. 79, pp. 233-241 (2008).

130. S. Vaidyanathan and K. Rajagopal, Global chaos synchronization of Lü and Pan systems by adaptive nonlinear control, Communications in Computer and Information Science, vol. 205, pp. 193-202 (2011).

131. V. Sundarapandian and R. Karthikeyan, Anti-synchronization of Lü and Pan chaotic systems by adaptive nonlinear control, European Journal of Scientific Research, vol. 64, pp. 94-106 (2011).

132. V. Sundarapandian and R. Karthikeyan, Anti-synchronization of hyperchaotic Lorenz and hyperchaotic Chen systems by adaptive control, International Journal of Systems Signal Control and Engineering Application, vol. 4, pp. 18-25 (2011).

133. V. Sundarapandian and R. Karthikeyan, Adaptive antisynchronization of uncertain Tigan and Li systems, Journal of Engineering and Applied Sciences, vol. 7, pp. 45-52 (2012).

134. P. Sarasu and V. Sundarapandian, Generalized projective synchronization of three-scroll chaotic systems via adaptive control, European Journal of Scientific Research, vol. 72, pp. 504-522 (2012).

135. P. Sarasu and V. Sundarapandian, Generalized projective synchronization of two-scroll systems via adaptive control, International Journal of Soft Computing, vol. 7, pp. 146-156 (2012).

136. P. Sarasu and V. Sundarapandian, Adaptive controller design for the generalized projective synchronization of 4-scroll systems, International Journal of Systems Signal Control and Engineering Application, vol. 5, pp. 21-30 (2012).

137. S. Vaidyanathan and K. Rajagopal, Global chaos synchronization of hyperchaotic Pang and hyperchaotic Wang systems via adaptive control, International Journal of Soft Computing, vol. 7, pp. 28-37 (2012).

138. S. Vaidyanathan and S. Pakiriswamy, Generalized projective synchronization of six-term Sundarapandian chaotic systems by adaptive control, vol. 6 (2), pp. 153-163 (2013).

139. S. Vaidyanathan and S. Pakiriswamy, Adaptive controller design for the generalized projective synchronization of circulant chaotic systems with unknown parameters, vol. 7 (1), pp. 55-74 (2014).

140. S.H. Lee, V. Kapila, M. Porfiri and A. Panda, Master-slave synchronization of continuously and intermittently coupled sampled-data chaotic oscillators, Communications in Nonlinear Science and Numerical Simulation, vol. 15 (12), pp. 4100-4113 (2010).

141. C.K. Zhang, L. Jiang, Y. He, Q.H. Wu and M. Wu, Asymptotical synchronization for chaotic Lur'e systems using sampled-data control, Communications in Nonlinear Science and Numerical Simulation, vol. 18, pp. 2743-2751 (2013).

142. X. Xiao, L. Zhou, and Z. Zhang, Synchronization of chaotic Lur'e systems with quantized sampled-data controller, Communications in Nonlinear Science and Numerical Simulation, vol. 19, pp. 2039-2047 (2014).

143. S. Rasappan and S. Vaidyanathan, Global chaos synchronization of WINDMI and Coullet chaotic systems by backstepping 
control, Far East Journal of Mathematical Sciences, vol. 67, pp. 265-287 (2012)

144. S. Rasappan and S. Vaidyanathan, Synchronization of hyperchaotic Liu system via backstepping control with recursive feedback, Communications in Computer and Information Science, vol. 305, pp. 212-221 (2012).

145. S. Rasappan and S. Vaidyanathan, Hybrid synchronization of nscroll Chua and Lur'e chaotic systems via backstepping control with novel feedback, Archives of Control Sciences, vol. 22, pp. 343-365 (2012).

146. R. Suresh and V. Sundarapandian, Global chaos synchronization of a family of $n$-scroll hyperchaotic Chua circuits using backstepping control with recursive feedback, Far East Journal of Mathematical Sciences, vol. 73, pp. 73-95 (2013).

147. S. Rasappan and S. Vaidyanathan, Hybrid synchronization of n-scroll chaotic Chua circuits using adaptive backstepping control design with recursive feedback, Malaysian Journal of Mathematical Sciences, vol. 7, pp. 219-246 (2013).

148. S. Vaidyanathan and S. Rasappan, Global chaos synchronization of n-scroll Chua circuit and Lur'e system using backstepping control design with recursive feedback, Arabian Journal for Science and Engineering, vol. 39, pp. 3351-3364 (2014).

149. S. Rasappan and S. Vaidyanathan, Global chaos synchronization of WINDMI and Coullet chaotic systems using adaptive backstepping control design, Kyungpook Mathematical Journal, vol. 54, pp. 293-320 (2014).

150. H.T. Yau, Chaos synchronization of two uncertain chaotic nonlinear gyros using fuzzy sliding mode control, Mechanical Systems and Signal Processing, vol. 22, pp. 408-418 (2008).

151. H. Li, X. Liao, C. Li, and C. Li, Chaos control and synchronization via a novel chatter free sliding mode control strategy, Neurocomputing, vol. 74, pp. 3212-3222 (2012).

152. S. Vaidyanathan and S. Sampath, Global chaos synchronization of hyperchaotic Lorenz systems by sliding mode control, Communications in Computer and Information Science, vol. 205, pp. 156-164 (2011).

153. V. Sundarapandian and S. Sivaperumal, Sliding controller design of hybrid synchronization of four-wing chaotic systems, International Journal of Soft Computing, vol. 6, pp. 224-231 (2011).

154. S. Vaidyanathan and S. Sampath, Anti-synchronization of fourwing chaotic systems via sliding mode control, International Journal of Automation and Computing, vol. 9, pp. 274-279 (2012).

155. H.T. Yau, Chaos synchronization of two uncertain chaotic nonlinear gyros using fuzzy sliding mode control, Mechanical Systems and Signal Processing, vol. 22(2), pp. 408-418 (2013).

156. S. Vaidyanathan, Global chaos synchronisation of identical Li$\mathrm{Wu}$ chaotic systems via sliding mode control, International Journal of Modelling, Identification and Control, vol. 22, no. 2, pp. 170-177 (2014).

157. S. Vaidyanathan and A.T. Azar, Anti-synchronization of identical chaotic systems using sliding mode control and an application to Vaidyanathan-Madhavan chaotic systems, Studies in Computational Intelligence, vol. 576, pp. 527-547 (2015).

158. S. Vaidyanathan and A.T. Azar, Hybrid synchronization of identical chaotic systems using sliding mode control and an application to Vaidyanathan chaotic systems, Studies in Computational Intelligence, vol. 576, pp. 549-569 (2015).

159. O.E. Rössler, An equation for hyperchaos, Physics Letters A, vol. 71, pp. 155-157 (1979).
160. Q. Jia, Hyperchaos generated from the Lorenz chaotic system and its control, Physics Letters A, vol. 366, pp. 217-222 (2007).

161. A. Chen, J. Lu, J. Lü and S. Yu, Generating hyperchaotic Lü attractor via state feedback control, Physica A, vol. 364, pp. 103110 (2006).

162. X. Li, Modified projective synchronization of a new hyperchaotic system via nonlinear control, Communications in Theoretical Physics, vol. 52, pp. 274-278 (2009).

163. J. Wang and Z. Chen, A novel hyperchaotic system and its complex dynamics, International Journal of Bifurcation and Chaos, vol. 18, pp. 3309-3324 (2008).

164. D. Ghosh and S. Bhattacharya, Projective synchronization of new hyperchaotic system with fully unknown parameters, Nonlinear Dynamics, vol. 61, pp. 11-21 (2010).

165. S. Vaidyanathan, A ten-term novel 4-D hyperchaotic system with three quadratic nonlinearities and its control, International Journal of Control Theory and Applications, vol. 6 (2), pp. 97109 (2013).

166. S. Vaidyanathan, Qualitative analysis and control of an eleventerm novel 4-D hyperchaotic system with two quadratic nonlinearities, International Journal of Control Theory and Applications, vol. 7 (1), pp. 35-47 (2014).

167. S. Vaidyanathan, Ch.K. Volos and V. -T. Pham, Hyperchaos, adaptive control and synchronization of a novel 5-D hyperchaotic system with three positive Lyapunov exponents and its SPICE implementation, Archives of Control Sciences, vol. 24 (4), pp. 409-446 (2014).

168. T. Rikitake, Oscillations of a system of disk dynamos, Mathematical Proceedings of the Cambridge Philosophical Society, vol. 54 (1), pp. 89-105 (1958).

169. A.E. Cook and P.H. Roberts, The Rikitake two disk dynamo system, Mathematical Proceedings of the Cambridge Philosophical Society, vol. 68 (2), pp. 547-569 (1970).

170. Z. Wang, S. Cang, E.O. Ochola and Y. Sun, A hyperchaotic system without equilibrium, Nonlinear Dynamics, vol. 69, pp. 531-537 (2012).

171. V. - T. Pham, F. Rahma, M. Frasca and L. Fortuna, Dynamics and synchronization of a novel hyperchaotic system without equilibrium, International Journal of Bifurcation and Chaos, vol. 24 (6), art. no. 1450087 (2014).

172. V. -T. Pham, Ch. K. Volos and L.V. Gambuzza, A memristive hyperchaotic system without equilibrium, Scientific World Journal, vol. 2014, art no. 368986 (2014).

173. S. Jafari and J.C. Sprott, Simple chaotic flows with a line equilibrium, Chaos, Solitons and Fractals, vol. 57, pp. 79-84 (2013).

174. G.A. Leonov, N.V. Kuznetsov, O.A. Kuznetsova, S.M. Seledzhi and V.I. Vagaitsev, Hidden oscillations in dynamical systems, WSEAS Transactions on Systems and Control, vol. 6 (2), pp. 54-67 (2011).

175. G.A. Leonov, N.V. Kuznetsov and V.I. Vagaitsev, Localization of hidden Chua's attractors, Physics Letters A, vol. 375 (23), pp. 2230-2233 (2011).

176. G.A. Leonov and N.V. Kuznetsov, "Hidden attractors in dynamical systems: from hidden oscillations in HilbertKolmogorov, Aizerman, and Kalman problems to hidden chaotic attractor in Chua circuits, International Journal of Bifurcation and Chaos, vol. 23 (1), art. no. 1330002 (2013).

177. H.K. Khalil, Nonlinear System, $3^{\text {rd }}$ ed., Prentice Hall, New Jersey, USA (2002). 